\title{
Protein Replacement Therapy
}

National Cancer Institute

\section{Source}

National Cancer Institute. Protein Replacement Therapy. NCI Thesaurus. Code C16221.

Administration of a protein to compensate for the loss, as from disease or surgery, of a gland or tissue that would normally produce the protein. 